\title{
The FIREBIRD-II CubeSat mission: Focused investigations of relativistic electron burst intensity, range, and dynamics
}

Cite as: Rev. Sci. Instrum. 91, 034503 (2020); https://doi.org/10.1063/1.5137905

Submitted: 13 November 2019 . Accepted: 03 March 2020 . Published Online: 19 March 2020

A. T. Johnson (D), M. Shumko (D), B. Griffith (D), D. M. Klumpar, J. Sample (D), L. Springer, N. Leh, H. E. Spence (D), S. Smith (D), A. Crew (D), M. Handley (D) K. M. Mashburn (D), B. A. Larsen (D), and J. B. Blake (iD)

\section{ARTICLES YOU MAY BE INTERESTED IN}

An improved adaptive signal averaging technique for noise reduction and tracking enhancements in continuous wave magnetic resonance

Review of Scientific Instruments 91, 033106 (2020); https://doi.org/10.1063/1.5123600

Direct measurement of 1-mN-class thrust and 100-s-class specific impulse for a CubeSat propulsion system

Review of Scientific Instruments 91, 035116 (2020); https://doi.org/10.1063/1.5121411

Direct frequency domain fluorescence lifetime imaging using field programmable gate arrays for real time processing

Review of Scientific Instruments 91, 033708 (2020); https://doi.org/10.1063/1.5127297

Lock-in Amplifiers

Find out more today

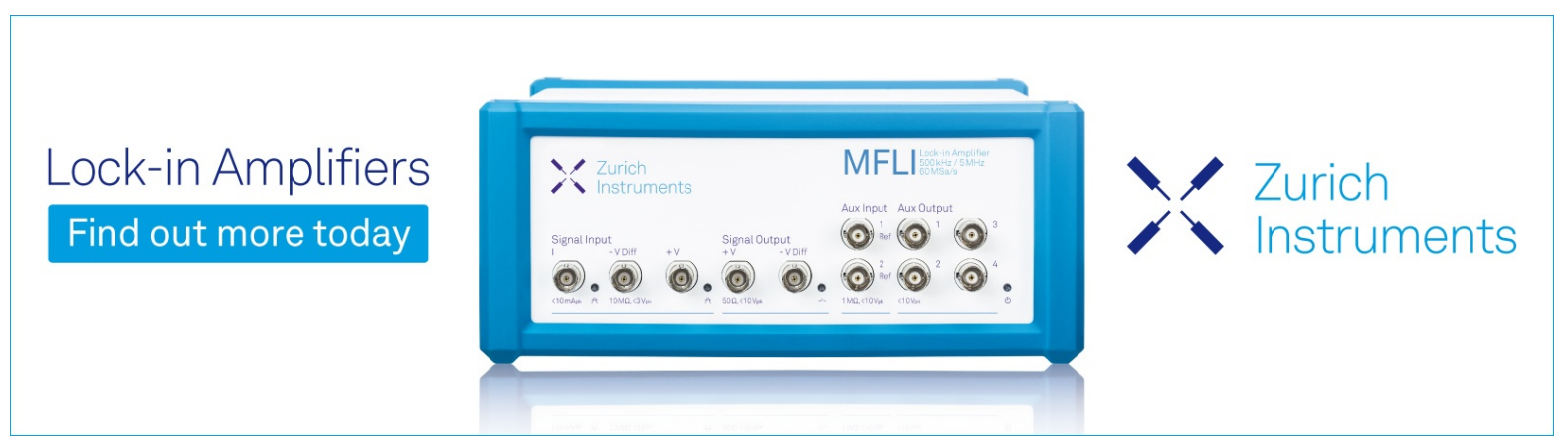

Rev. Sci. Instrum. 91, 034503 (2020); https://doi.org/10.1063/1.5137905 


\title{
The FIREBIRD-II CubeSat mission: Focused investigations of relativistic electron burst intensity, range, and dynamics
}

Cite as: Rev. Sci. Instrum. 91, 034503 (2020); doi: 10.1063/1.5137905

Submitted: 13 November 2019 - Accepted: 3 March 2020 •

Published Online: 19 March 2020
A. T. Johnson,
M. Shumko,
B. Griffith, ${ }^{1}$
D. M. Klumpar,' J. Sample,
L. Springer, ' N. Leh,
H. E. Spence, ${ }^{2}$
S. Smith, ${ }^{2}$ A. Crew,
M. Handley, ${ }^{4}$
K. M. Mashburn,
B. A. Larsen,
and J. B. Blake

\author{
AFFILIATIONS \\ ${ }^{1}$ Physics Department, Montana State University, Bozeman, Montana 59717, USA \\ ${ }^{2}$ Physics Department, University of New Hampshire, Durham, New Hampshire 03824, USA \\ ${ }^{3}$ Applied Physics Laboratory, Johns Hopkins University, Laurel, Maryland 20723, USA \\ “Los Alamos National Laboratory, Los Alamos, New Mexico 87545, USA \\ ${ }^{5}$ Space Science Applications Laboratory, The Aerospace Corporation, El Segundo, California 90245, USA
}

${ }^{a)}$ Author to whom correspondence should be addressed: arlotjohnson@gmail.com

\begin{abstract}
FIREBIRD-II is a National Science Foundation funded CubeSat mission designed to study the scale size and energy spectrum of relativistic electron microbursts. The mission consists of two identical $1.5 \mathrm{U}$ CubeSats in a low earth polar orbit, each with two solid state detectors that differ only in the size of their geometric factors and fields of view. Having two spacecraft in close orbit allows the scale size of microbursts to be investigated through the intra-spacecraft separation when microbursts are observed simultaneously on each unit. Each detector returns high cadence ( $10 \mathrm{~s}$ of $\mathrm{ms}$ ) measurements of the electron population from $200 \mathrm{keV}$ to $>1 \mathrm{MeV}$ across six energy channels. The energy channels were selected to fill a gap in the observations of the Heavy Ion Large Telescope instrument on the Solar, Anomalous, and Magnetospheric Particle Explorer. FIREBIRD-II has been in orbit for 5 years and continues to return high quality data. After the first month in orbit, the spacecraft had separated beyond the expected scale size of microbursts, so the focus has shifted toward conjunctions with other magnetospheric missions. FIREBIRD-II has addressed all of its primary science objectives, and its long lifetime and focus on conjunctions has enabled additional science beyond the scope of the original mission. This paper presents a brief history of the FIREBIRD mission's science goals, followed by a description of the instrument and spacecraft. The data products are then discussed along with some caveats necessary for proper use of the data.
\end{abstract}

Published under license by AIP Publishing. https://doi.org/10.1063/1.5137905

\section{SCIENCE OVERVIEW}

FIREBIRD is a pair of $1.5 \mathrm{U}\left(10 \times 10 \times 15 \mathrm{~cm}^{3}\right)$ CubeSats (Fig. 1) supported by the National Science Foundation (NSF) and designed to investigate the spatial scale and energy dependence of electron microbursts. ${ }^{1,2}$ Microbursts are short intensifications of electron precipitation into the atmosphere lasting up to a few hundred milliseconds. An example of microbursts observed by Flight Unit 4 on December 23, 2016, is shown in Fig. 2. The term "microburst" was first used by Anderson and Milton ${ }^{3}$ to describe enhancements in balloon observations of $\leq 100 \mathrm{keV}$ bremsstrahlung $\mathrm{x}$ rays caused by electrons impacting the atmosphere. Later, balloon observations up to $300 \mathrm{keV}$ revealed microbursts to be a significant loss process in the dayside magnetosphere. ${ }^{4}$ More recently, relativistic (>1 MeV) electron microbursts have been observed in situ by spacecraft. ${ }^{5-7}$ Despite the historical record of observations, much is still unknown about microbursts. FIREBIRD was designed to answer three outstanding questions of microburst science:

1. What is the spatial scale size of an individual microburst?

2. What is the energy dependence of an individual microburst?

3. How much total electron loss do microbursts produce globally? 


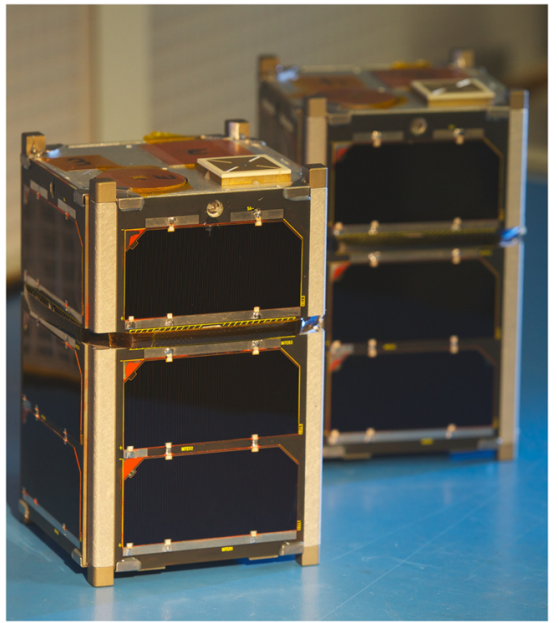

FIG. 1. The FIREBIRD-II CubeSats fully assembled before delivery with Flight Unit 4 in front. The top third of each flight unit contains the FIRE (FIREBIRD Instrument for Relativistic Electrons) instrument while the bottom two thirds contain the BIRD (Bus In support of Radiation Detector) spacecraft bus. The antenna can be seen wrapped around the gap between the two sections. The top of the spacecraft has the global positioning system (GPS) antenna (square seen to the lower right) and the two detectors protected by dust covers removed before launch. The surface detector is under the square cover at the top right and the collimated detector under the circular cover to the bottom left.

The spatial scale size of microbursts has been investigated since their discovery and is an important factor toward understanding the significance of microbursts as a loss process. Balloons flown by Parks $^{8}$ measured bremsstrahlung $\mathrm{x}$ rays resulting from electron

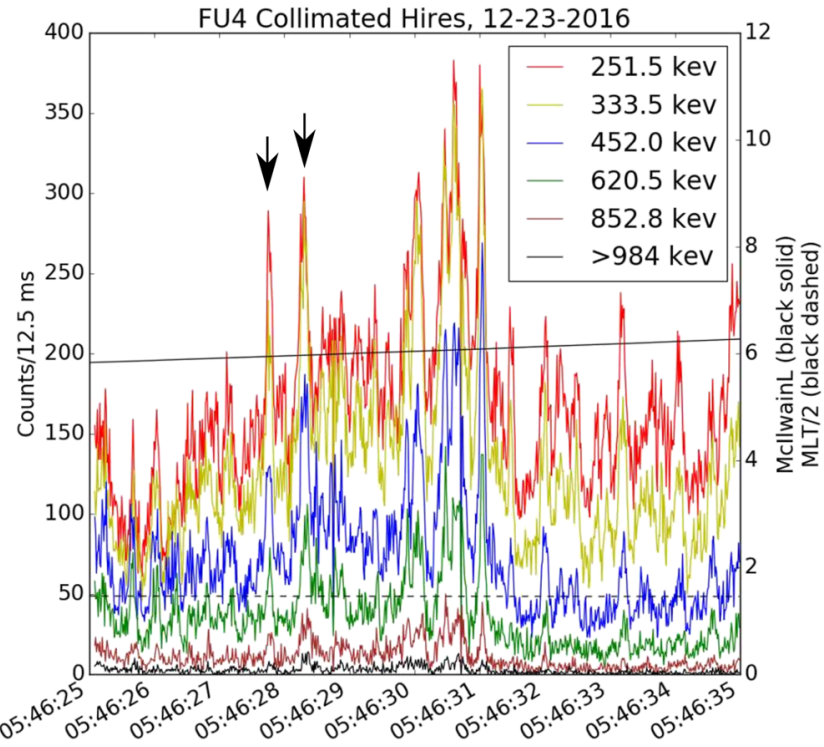

FIG. 2. Microbursts observed by FU4 on December 23, 2016. Microbursts often arrive in a "train" of several bursts and are seen across the full energy range of the instrument. The arrows indicate two of the microbursts observed. precipitation. The balloon payload consisted of four collimated $\mathrm{x}$ ray detectors: one pointing at zenith with the other three at $30^{\circ}$ from zenith and $120^{\circ}$ apart in azimuth. Parks ${ }^{8}$ estimated a microburst radius of $40 \pm 14 \mathrm{~km}$; however, events were only considered when seen by all four telescopes placing a minimum size on an event to be considered. Parks ${ }^{8}$ notes that most bursts were seen by at least two telescopes, but many were only seen by the zenith telescope placing a maximum radius on those bursts of $20 \mathrm{~km}$. Blake et al. . $^{6}$ found a bouncing packet of microburst electrons on the Solar, Anomalous, and Magnetospheric Particle EXplorer (SAMPEX) Heavy Ion Large Telescope (HILT) detector, which must have been "at least a few tens of kilometers." Blake et al. ${ }^{6}$ found few events where the entire decay of a microburst could be observed and concluded that a typical microburst has a scale size of less than a few tens of gyroradii, on the order of a few kilometers. Dietrich et al. ${ }^{9}$ used SAMPEX data, along with ground-based very low frequency stations, to conclude that the microbursts observed during one SAMPEX pass had scale sizes less than $4 \mathrm{~km}$.

Another important factor toward understanding microbursts is the energy spectrum. Comparing the energy spectrum of a microburst to the background energy spectrum in the radiation belts gives crucial insight into the processes that scatter microburst electrons and helps determine the importance of microbursts as a loss process at various energies. Early balloon observations recorded microbursts up to $300 \mathrm{keV}$, but to date, no microbursts above a few hundred keV have been observed by balloons. ${ }^{10,11}$ Spacecraft have observed relativistic microbursts, but the energy range from a few hundred $\mathrm{keV}$ to $1 \mathrm{MeV}$ has not been well studied and may be crucial. Blake et al. ${ }^{6}$ compared microburst detections on the $150 \mathrm{keV}$ and $>1 \mathrm{MeV}$ channels of the HILT detector on SAMPEX and found that they were not always correlated. This could indicate a difference in the generation mechanism for these energies and a possible break in the microburst energy spectrum.

The final objective is to determine the importance of microburst electron loss globally. Using storm time SAMPEX data, it has been estimated that microbursts are capable of emptying the outer radiation belt of $1 \mathrm{MeV}$ electrons on the order of a day. ${ }^{12-14}$ The largest source of error in the SAMPEX loss estimates is the limited Magnetic Local Time (MLT) coverage, ${ }^{12,13}$ requiring an assumption in the spatial extent of the microburst region and the activity levels across that region. The scale size and energy dependence of microbursts are also important elements in this estimation. It is not expected that FIREBIRD alone will be able to fully address this question, but by combining the FIREBIRD data with other radiation belt missions, many of the underlying assumptions in this calculation can be explored and quantified.

\section{THE FIREBIRD CUBESATS}

CubeSats are a relatively new scientific tool that offers low-cost access to space. Early CubeSats were regarded as educational tools: useful for introducing new scientists and engineers to spacecraft but offering little scientific merit. Since then, CubeSats have proven their ability to return novel observations and form the basis for highprofile science despite the limited budget and scope associated with a CubeSat mission. ${ }^{15,16}$ As of March 2018, the NSF had flown 20 scientific CubeSats across 14 missions. 
CubeSat missions related to FIREBIRD, such as the Colorado Student Space Weather Experiment (CSSWE) ${ }^{18}$ and AeroCube-6 (AC6) ${ }^{19}$ have proven the utility of the CubeSat platform for studying particle precipitation from the radiation belts. CSSWE was a 3 U CubeSat developed by the University of Colorado Boulder, which was launched on September 13, 2012, and operated for just under 2 years measuring electron and proton flux. CSSWE is one of the most successful CubeSat missions to date with over 19 peer reviewed publications $^{17}$ (e.g., Ref. 20). AC6 was a pair of 0.5 U CubeSats developed by The Aerospace Corporation and launched on June 19, 2014. AC6 contained multiple instruments, but of interest to the space weather community are three Aerospace-Teledyne microdosimeters that measure the integral electron flux. A unique feature of AC6 was the ability to control the orbital separation of the spacecraft by changing the drag force on each, which led to the discovery of latitudinally narrow persistent spatial precipitation structures. ${ }^{21}$ FIREBIRD improves on these missions with increased time and energy resolution, which is critical to the study of microbursts.

FIREBIRD was developed in collaboration between several institutes. Each FIREBIRD unit is made up of a payload known as FIRE (FIREBIRD Instrument for Relativistic Electrons), which was developed at the University of New Hampshire (UNH), and a bus known as BIRD (Bus In support of Radiation Detector), which was developed at Montana State University (MSU). A key component of FIRE is the DAPPER (Dual Amplifier Pulse Peak Energy Rundown) chip provided by The Aerospace Corporation. Preliminary GEANT (GEometry ANd Tracking) ${ }^{22,23}$ modeling and magnetic field mapping for data processing during the early mission was performed at Los Alamos National Laboratory (LANL).

There are two primary FIREBIRD missions, FIREBIRD-I consisting of Flight Unit 1 and Flight Unit 2 and FIREBIRD-II consisting of Flight Unit 3 and Flight Unit 4. FIREBIRD-I was launched on December 6,2013 , into a $121^{\circ}$ inclination orbit. Flight Unit 1 was unresponsive immediately following launch, but contact was made in April and lasted into September. Flight Unit 2 was operational immediately following launch but only operated for 6 weeks, meaning the two units were never on simultaneously, preventing much of the primary science. In addition, the orbital inclination kept the satellites from reaching the latitude of the outer radiation belt on many of their orbits. When the satellites did reach the outer belt, it was at a local time where microbursts are not typically observed. The combination of these factors resulted in FIREBIRD-I observing no verified microbursts during its lifetime.

FIREBIRD-II is a follow-on mission to FIREBIRD-I with identical instrumentation and improved satellite systems. It was determined that the short lifetime of FIREBIRD-I was primarily due to the power system. During the development of FIREBIRD-II, an updated power system was built in house with a focus on simplicity and robustness (see Sec. IV for more detail). FIREBIRD-II was launched on January 31,2015 , into a $99.1^{\circ}$ inclination orbit, and both units were operational an hour following launch. FIREBIRD-II has now been in orbit for five years. Flight Unit 3 (FU3) had a battery failure in November 2019 preventing further science collection but continues to operate in an engineering mode. FU4 continues to operate and return high quality data. For the remainder of this paper, "FIREBIRD" will refer to FIREBIRD-II unless otherwise noted.

The primary design requirements of FIREBIRD can be directly linked to the science questions it seeks to answer. FIREBIRD addresses the scale size of microbursts by having a pair of observations in close proximity. If a microburst is observed by both flight units simultaneously, it must be larger than the separation between the two units. Both flight units began taking data an hour after release from the launch vehicle, corresponding to a separation of less than $10 \mathrm{~km}$ to make this measurement. The FIREBIRD energy range was chosen to probe the energy gap between the SAMPEX HILT energies, with five energy channels ranging from $200 \mathrm{keV}$ to $1 \mathrm{MeV}$ and a sixth $>1 \mathrm{MeV}$ channel. Addressing the total electron loss globally did not drive any additional design considerations but underlines the importance of combining FIREBIRD observations with other sources to present a more complete picture of microburst dynamics. Significant focus has been placed on magnetic conjunctions between FIREBIRD and other magnetospheric missions.

FU3 Hi-Res October 11, 2018
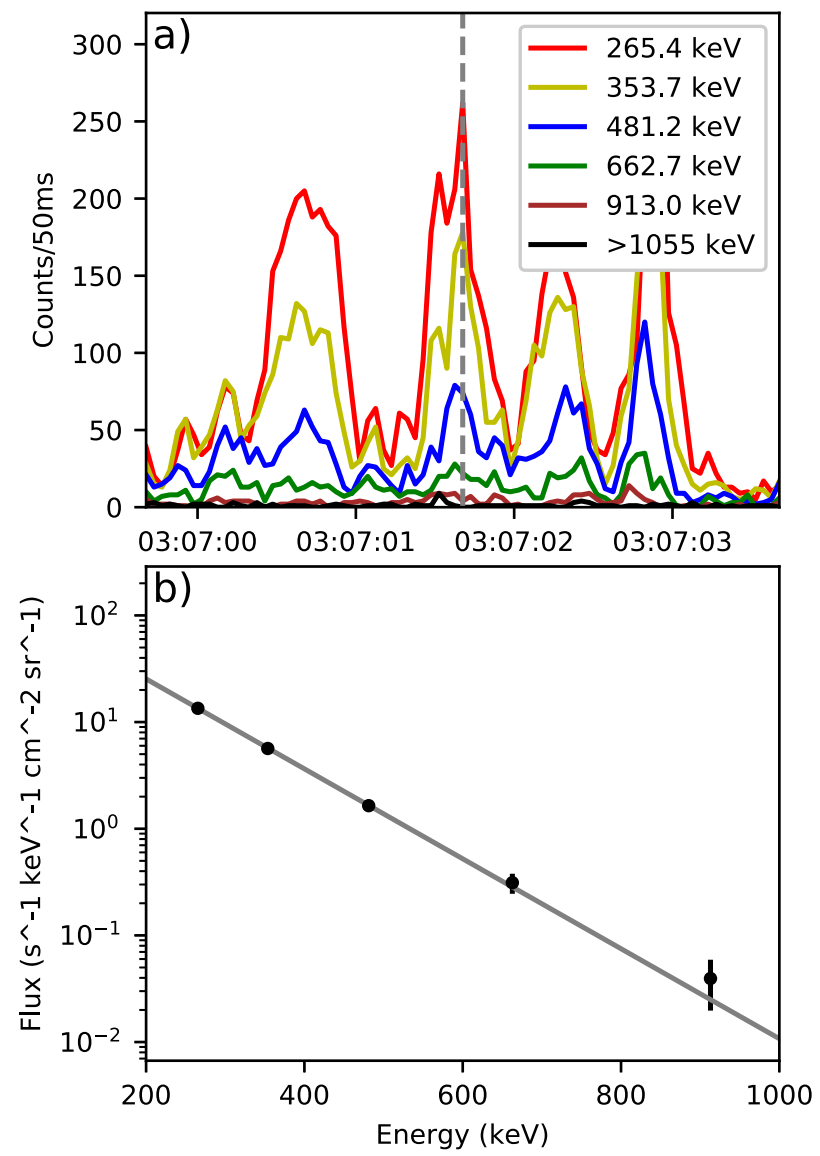

FIG. 3. An example of the energy spectrum of a microburst observed by FU3 on October 11, 2018. (a) Data from the collimated detector on FU3. The vertical dashed gray line indicates the microburst being analyzed. (b) Modeled electron flux for this microburst. The solid gray line is the modeled flux function, assumed to be e-folding, found using the GEANT mass model (see Sec. $V$ for more detail). The points are found by dividing the observed counts by an effective geometric factor calculated from the modeled flux function. The error bars are calculated as $\sqrt{N}$ counts and then scaled by the effective geometric factor. 
FIREBIRD has been successful in addressing each of its science objectives. Crew et al ${ }^{24}$ identified microbursts observed simultaneously on each FIREBIRD unit at a separation of $11 \mathrm{~km}$. This implies that the spatial size of those microbursts must have been at least $11 \mathrm{~km}$ and represents the first direct measurement of a microburst's scale size. Shumko et al. ${ }^{25}$ identified a bouncing packet of microburst electrons observed by both FIREBIRD units and calculated a minimum latitudinal scale size of $30 \mathrm{~km}$ based on satellite position and a longitudinal scale size of $50 \mathrm{~km}$ using drift time analysis. FIREBIRD has observed thousands of microbursts at a variety of geomagnetic conditions, allowing an in depth look at the spectral properties of microbursts, which is currently underway. An example microburst spectrum from this study is presented in Fig. 3. Using FIREBIRD data combined with AC6 and the Balloon Array for Radiation Belt Storm Probes (RSBPs) (Van Allen Probes) Relativistic Electron Losses (BARREL), ${ }^{26}$ Anderson et al. ${ }^{27}$ was able to determine the spatial scale and duration of a particular microburst region, which helps constrain the global microburst loss estimates.

FIREBIRD has also contributed to additional science beyond its primary objectives. Another objective of microburst science has been to identify the mechanism that scatters microburst electrons into the loss cone. The most likely candidate is whistler mode chorus ${ }^{28}$ that have similar temporal and spatial characteristics with microbursts. ${ }^{7,29-32}$ Using a close conjunction between Van Allen Probe $\mathrm{A}^{33}$ and FU4, Breneman et al. ${ }^{34}$ demonstrated a direct link between chorus wave activity and microburst generation. FIREBIRD has also contributed to magnetospheric science beyond microbursts. Capannolo et al. ${ }^{35}$ used FIREBIRD in conjunction with Van Allen Probe A and other nearby wave measurements to study the electron precipitation caused by Electromagnetic ion cyclotron (EMIC) waves. In addition, there are ongoing investigations using FIREBIRD data into the effect of electron precipitation on atmospheric chemistry and the signature of microbursts when viewed from the Poker Flat Incoherent Scatter Radar (PFISR). ${ }^{36}$ New FIREBIRD data are uploaded daily to http://solar.physics.montana.edu/FIREBIRD_II/.

\section{DETECTORS}

The FIRE instrument package was developed at the University of New Hampshire, containing two detectors referred to as the collimated and surface detectors. Each detector is a $1500 \mu \mathrm{m}$ thick, $32 \mathrm{~mm}$ diameter silicon solid-state detector, which were acquired as surplus from a previous National Oceanic and Atmospheric Administration (NOAA) mission. Each detector can fully absorb electrons up to $1050 \mathrm{keV}$ above which some penetration will occur. The detectors are set inside an aluminum housing with an aluminum foil covering the entrance to make the housing light tight and stop low energy particles. Particles in the energy range of interest lose approximately $25 \mathrm{keV}$ going through the foil, which is negligible compared to the $200 \mathrm{keV}$ minimum energy. The collimated detector has an additional aluminum collimator above the housing, which reduces the angular response and geometric factor of that detector. Figure 4 shows a FIREBIRD unit during integration and testing (top) and one of the detectors in an opened collimated housing (bottom). The angular response and geometric factor are described in detail in Sec. V.

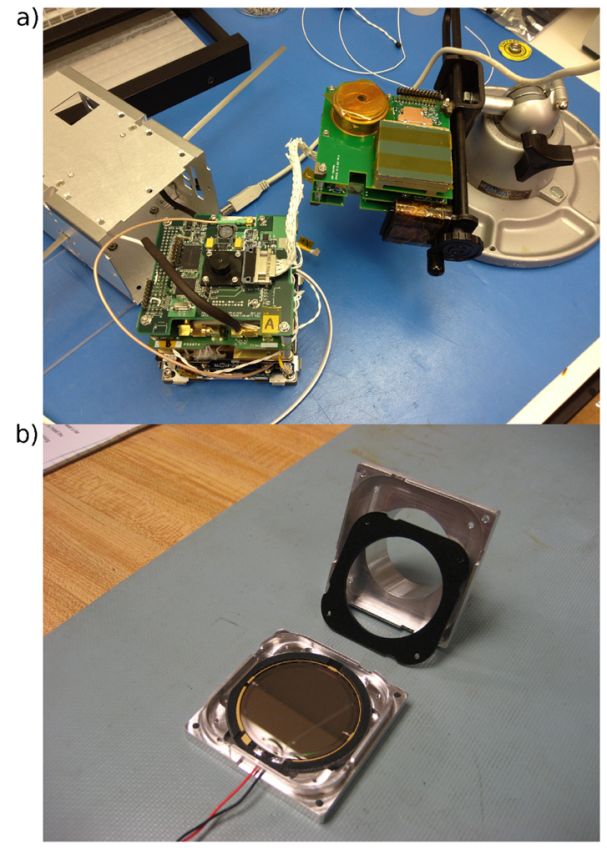

FIG. 4. (a) One of the FIREBIRD-I units during integration and testing. The spacecraft bus is the stack of boards resting on the table, and the instrument boards are held in the clamp. The detectors are within the covered housings protruding from the instrument board, with the collimated detector at the upper left (circular) and surface detector at the bottom right (square). The instrument boards from top to bottom are the analog board, digital board, and power board. (b) A detector inside a collimated housing. The collimator is visible as the top piece of the housing. The aluminum foil (not shown) is placed between the delrin ring and the top of the housing.

The initial pulse processing is done using a DAPPER (Dual Amplifier Pulse Peak Energy Rundown) chip that was provided by The Aerospace Corporation (see Sec. 4 of Ref. 37), originally developed for use in the Fly's Eye Energetic Particle Spectrometer (FEEPS) instrument on the Magnetospheric MultiScale (MMS) mission. ${ }^{37}$ The DAPPER creates a fixed-height, variable width pulse with a duration linearly proportional to the input signal from the detector. This pulse is then timed by a Field-Programmable Gate Array (FPGA) on the digital board and mapped into a digital bin ranging from 0 to 255 . Due to telemetry constraints, the 256 digital channels are down-sampled to six energy channels, which make up the high resolution data product (see Sec. VI). The borders between the six energy channels are on-orbit configurable, allowing portions of the energy spectrum to be investigated in fine resolution.

The DAPPER has an on-board test pulser that can be used to assess instrument health. During a pulser test, FIREBIRD steps through the digital channels to sample the response across the energy range. The signal appears as a Gaussian peak with a measurable Full Width at Half Maximum (FWHM) and peak location. The test pulser was utilized for instrument testing on each unit before launch and has been run twice in orbit, once in November 2017 and again in September 2018 (Fig. 5). Additional pulser tests are planned to be run each year to monitor any changes in detector response. 

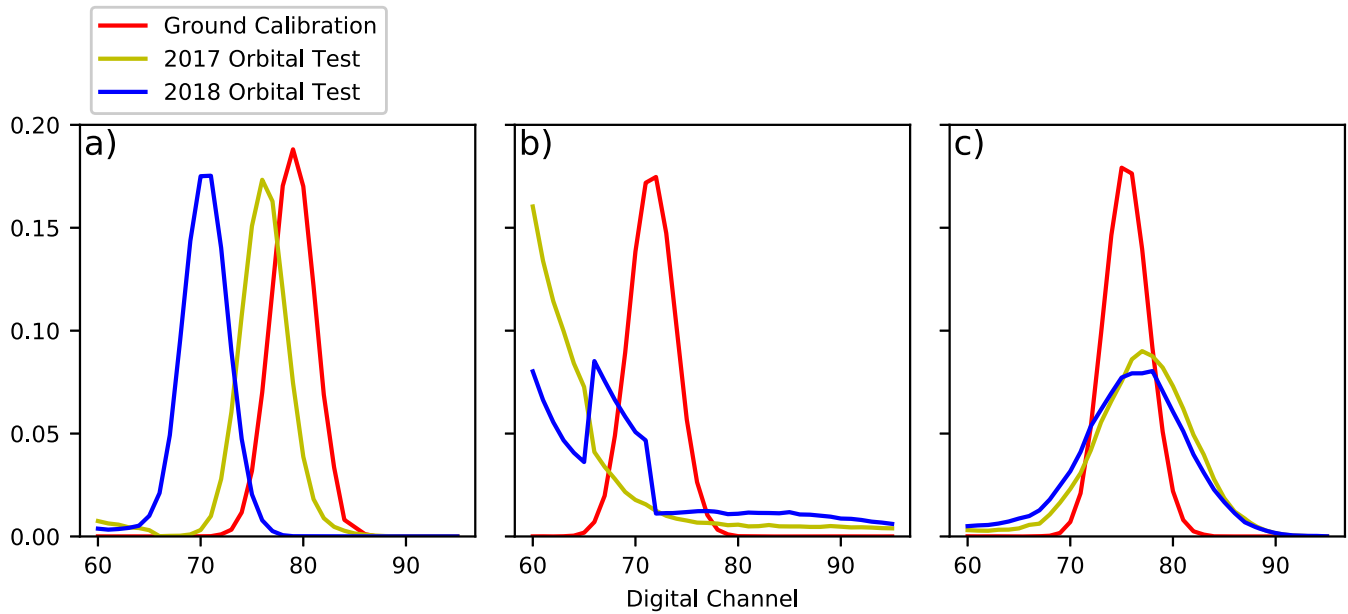

FIG. 5. Comparison of the test pulser runs performed on FIREBIRD-II. Each curve has been normalized such that its integral is unity. The FU3 collimated detector (a) shows a shift in the peak location indicating a shift in the gain. The FU3 surface detector (b) shows a large shift in gain, and much of the orbital test falls in digital bins that were not sampled. The precise status of the surface detector is unknown, but it is clear that it no longer functions as intended. The FU4 collimated detector (c) shows a similar peak location but broader peak width, indicating a similar gain but increased detector noise. The surface detector on FU4 has not functioned since launch.

A shift in the peak location, such as observed on the FU3 collimated detector [Fig. 5(a)], indicates the gain of the digital channels has shifted, causing each bin to contain a different energy than initially calibrated. An increase in peak width, such as observed on the FU4 collimated detector [Fig. 5(c)], indicates an increase in detector noise. The surface detector on FU3 [Fig. 5(b)] has seen a large change in the test pulser response. Most of the orbital test data fell into digital bins that were not sampled for the test, so the precise nature of the detector is unknown; however, it is clear from the pulser tests that it no longer functions as intended. A qualitative change in the FU3 surface time series data was first noticed in late July 2015, near the end of campaign 4 (see Sec. VII), but the detector performed as expected for the first four campaigns. The surface detector on FU4 has not functioned since launch.

\section{SPACECRAFT SYSTEMS}

The spacecraft structure and bus were assembled largely from Commercial Off The Shelf (COTS) hardware by Montana State University. The structure is a modified Pumpkin, Inc., $1.5 \mathrm{U}$ chassis with solar panels mounted to each of the $10 \times 15 \mathrm{~cm}^{2}$ faces with a smaller panel attached to the bottom of the satellite. For FIREBIRD-I, the solar panels were designed and assembled in-house at MSU; however, this proved to be very labor intensive, so FIREBIRD-II used professionally assembled panels from Vanguard Electronics.

The spacecraft bus primarily consists of four boards: Command and Data Handling (CDH), Communications (COMM), Multi-Function Interface Board (MFIB), and Electrical Power System (EPS). The CDH from Pumpkin, Inc., is a Motherboard and Pluggable Processor Module that controls all subsystems on the spacecraft and stores and executes on-orbit configurable Command Sequences (CMDSEQs). The COMM transceiver is obtained from Astronautical Development, LLC. He-100 radio with a VHF uplink and an ultrahigh frequency (UHF) downlink and operates in the
Amateur radio bands. The radio is connected to a monopole antenna for each band made from spring steel tape measures. The MFIB was designed and built at MSU and acts as an interface to the FIRE payload and any other subsystem that could not be directly connected to one of the COTS subsystems. The MFIB also features a PIC24F microcontroller, a 2 GB NAND flash for FIRE data processing and storage, and an OEMV1 GPS receiver from NovAtel, Inc., for synchronizing the Real Time Clock (RTC) on the CDH.

The CDH, COMM, and MFIB boards flew on both FIREBIRDI and FIREBIRD-II with some minor adjustments based on lessons learned from the previous mission. The EPS on FIREBIRD-I was developed by a commercial aerospace partner considering entering the CubeSat market. The EPS was designed for a higher electrical load, such as those of a $3 \mathrm{U}$ or $6 \mathrm{U}$ CubeSat and was, therefore, very inefficient under FIREBIRD-I's smaller loads. Flaws in this board led to the batteries being overcharged when the satellites had precessed into a full-sun orbit leading to battery degradation and an increased number of resets under high load. This was the primary cause of FIREBIRD-I's short lifetime. For FIREBIRD-II, a new EPS was designed and built at MSU based on the first MSU satellite, the Hiscock Radiation Belt Explorer, ${ }^{38}$ which remained operational for several years. The new EPS is based on a Direct Energy Transfer system where the batteries are directly connected to the solar arrays via a COTS battery protection circuit. When the batteries are fully charged and the solar panels are providing sufficient power, the batteries are disconnected from the system to prevent over-charging, and the solar panels will power the entire spacecraft. If the system power consumption increases, such as during radio transmission, or the power from the panels decrease, the batteries are reconnected to power the system.

Another key feature of the new EPS is a hardware WatchDog Timer (WDT), which power cycles the entire spacecraft every $12 \mathrm{~h}$. By resetting every $12 \mathrm{~h}$, any single event upsets due to radiation strikes or software bugs can be resolved without intervention from 
the ground. There have been several instances where the spacecraft have latched up and been unresponsive to ground commands, but in each case, the spacecraft returned to normal functionality after the WDT was triggered. The WDT has been an important factor in the longevity of FIREBIRD-II.

The $\mathrm{CDH}$ flight software is implemented in the C language in the $\mu \mathrm{C} / \mathrm{OS}$-II operating system from Micrium, Inc. The software was designed to be highly modular and configurable and achieves this primarily through leveraging the built-in command sequencer. Each CMDSEQ is a list of instructions for the spacecraft, which can be executed manually via radio uplink, called by other command sequences, or triggered by telemetry alarms generated by the telemetry monitor module. On each boot, the first CMDSEQ is executed, which then configures the spacecraft by calling other configuration CMDSEQs. Each CMDSEQ can be fully modified on-orbit if a flaw is found or to adjust parameters to better meet science goals (see Sec. VII A).

\section{DETECTOR RESPONSE MODELING IN GEANT4}

A FIREBIRD mass model has been created in GEANT4 for purposes of modeling detector response. GEANT uses Monte Carlo methods to simulate particle interactions and propagation through matter. ${ }^{22,23}$ The mass model was reconstructed from the final mechanical drawings of FIREBIRD. The detectors and elements near the detectors were modeled with high fidelity, while elements further away from the detectors were modeled as blocks of material that approximate the material properties and geometry of the spacecraft.

Electrons with a random energy according to a $1 / x$ distribution between $200 \mathrm{keV}$ and $2 \mathrm{MeV}$ are launched from a random location on a $25 \mathrm{~cm}$ radius sphere centered between the two detectors. The launch angle is selected according to a cosine distribution to create an isotropic flux within the source sphere. The initial conditions of each particle were determined using the Ranecu Random engine and General Particle Source GEANT packages. ${ }^{22,39}$ Each launched electron interacts with the FIREBIRD mass model, and the amount of energy deposited in the detector is calculated. The physical processes considered in the model were determined using the physics list recommended by the GEANT documentation for spacecraft analysis and particle energies typically found in the radiation belts.

For each electron launched in the simulation, the incident energy, incident angle, and energy deposited in the detector are recorded. The incident energies are binned into 99 logarithmically spaced bins with roughly equal counts due to the $1 / x$ energy distribution. The deposited energies in each incident energy bin are then binned by the FIREBIRD energy channel boundaries to determine the detector response. For each incident energy bin, the geometric factor for a FIREBIRD energy channel is

$$
G(E)=\frac{n(E)}{4 \pi^{2} r^{2} N_{i}},
$$

where $n(E)$ is the number of electrons deposited in FIREBIRD energy channel $E, r$ is the radius of the launch sphere, and $N_{i}$ is the total number of electrons launched within the incident energy bin. ${ }^{40}$
Figure 6(a) shows the energy dependent electron geometric factor for each of the energy bins on the FU3 collimated detector. With the exception of the $>1 \mathrm{MeV}$ channel, the geometric factor across the nominal energy range of each channel is approximately $6 \mathrm{~cm}^{2}$ sr, compared with an analytic geometric factor of $9 \mathrm{~cm}^{2}$ sr determined from the detector geometry. ${ }^{41}$ The FIREBIRD data product reports electron flux using the analytic geometric factors, but the
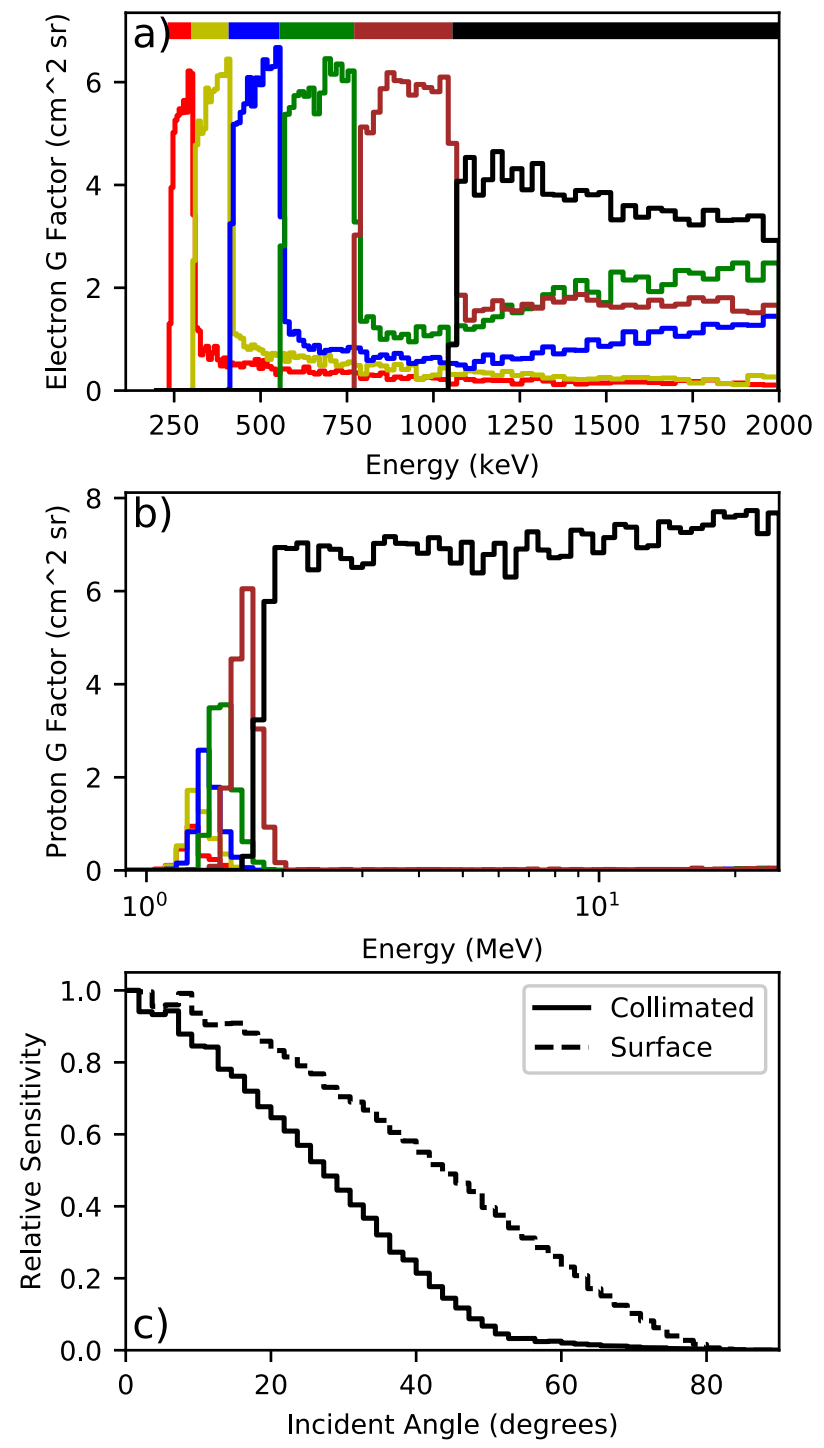

FIG. 6. A summary of GEANT modeling of the detectors aboard FU3. FU4 (not shown) has a similar geometric factor and angular response. (a) The energy dependent electron geometric factor calculated with GEANT for the collimated detector on FU3. The colored bar at the top indicates the nominal energy ranges. (b) The energy dependent proton geometric factor for the collimated detector on FU3. The differential channels are only sensitive to a narrow range of proton energies between $1 \mathrm{MeV}$ and $1.1 \mathrm{MeV}$. Proton contamination will only be present during periods of high energy proton flux and will predominantly appear in the integral channel. (c) Angular response of the FU3 detectors to mono-energetic $750 \mathrm{keV}$ electrons. 
TABLE I. Energy ranges of the FIREBIRD detectors (keV) and median geometric factors $\left(\mathrm{cm}^{2} \mathrm{sr}\right)$ during campaigns 1-20. The surface detector on FU4 has not functioned since launch, so its energy ranges are excluded. The response of the surface detector on FU3 changed significantly in July 2015 (see Sec. III), so the values shown here are only correct up until then.

\begin{tabular}{|c|c|c|c|c|c|}
\hline \multicolumn{2}{|c|}{ FU3 Collimated } & \multicolumn{2}{|c|}{ FU3 Surface } & \multicolumn{2}{|c|}{ FU4 Collimated } \\
\hline Energy range (keV) & $\mathrm{G}$ factor $\left(\mathrm{cm}^{2} \mathrm{sr}\right)$ & Energy range (keV) & $\mathrm{G}$ factor $\left(\mathrm{cm}^{2} \mathrm{sr}\right)$ & Energy range (keV) & $\mathrm{G}$ factor $\left(\mathrm{cm}^{2} \mathrm{sr}\right)$ \\
\hline $231.0-299.7$ & 5.4 & $176.4-240.9$ & 13.1 & $219.7-283.4$ & 5.5 \\
\hline $299.7-407.6$ & 5.8 & $240.9-342.2$ & 13.0 & $283.4-383.6$ & 5.4 \\
\hline $407.6-554.8$ & 6.0 & $342.2-480.3$ & 13.8 & $383.6-520.3$ & 5.7 \\
\hline $554.8-770.7$ & 5.8 & $480.3-683.0$ & 13.9 & $520.3-720.7$ & 5.9 \\
\hline $770.7-1055.2$ & 5.9 & $683.0-950.1$ & 14.1 & 720.7-985.0 & 5.8 \\
\hline$>1055.2$ & 3.8 & $>950.1$ & 11.2 & $>985.0$ & 4.2 \\
\hline
\end{tabular}

results of this modeling indicate the true flux in the collimated detector is about $50 \%$ higher than reported. The geometric factor of the surface detector (not shown) found in the GEANT model is approximately $14 \mathrm{~cm}^{2} \mathrm{sr}$, compared with an analytic geometric factor of $23 \mathrm{~cm}^{2}$ sr, indicating surface flux is about $60 \%$ higher than reported in the FIREBIRD data product. The median geometric factors in each energy channel are presented in Tables I and II.

Using the GEANT results, a flux spectrum can be modeled based on the count data reported by FIREBIRD. The flux is first estimated from the count data by dividing by an approximate geometric factor and the energy bin width. An assumed flux function, typically an e-folding or power law function, is then fit to these fluxes, integrated across the GEANT incident energy bins, and multiplied by the geometric factors calculated by Eq. (1) to model the counts that would be observed by FIREBIRD. The parameters of the flux function are then iterated to find the best agreement between the observed and modeled count rates.

The proton response was modeled in the same way as the electron response to investigate potential contamination. Figure 6(b) shows the energy dependent proton geometric factor in the collimated detector. The detector only reacts to protons above about $1 \mathrm{MeV}$; so, in most cases, proton contamination can be neglected. If high energy protons are encountered, the contamination will be mostly limited to the $>1 \mathrm{MeV}$ integral channel with the differential channels only responding to a narrow range of energies from $1 \mathrm{MeV}$ to $1.1 \mathrm{MeV}$.

The angular response of the FIREBIRD detectors has also been modeled. Monoenergetic electrons are launched from a random location on a $25 \mathrm{~cm}$ radius sphere in the same way as the model for geometric factor. Electrons that deposit at least $200 \mathrm{keV}$ into the detector are counted and binned by the launch incidence angle. The angular response was calculated for $250 \mathrm{keV}$ and $750 \mathrm{keV}$ electrons and yielded similar results. Figure 6(c) shows the angular response to $750 \mathrm{keV}$ electrons in the FU3 collimated and surface detectors. Both detectors show a soft cutoff with increasing angle. The angular response modeled in GEANT line up well with ground calibration of the instrument. The FWHM for $750 \mathrm{keV}$ electrons is calculated to be $61.8^{\circ}$ and $101.8^{\circ}$ for the collimated and surface detectors, respectively. The FWHM for $250 \mathrm{keV}$ electrons (not shown) is slightly smaller, at $58.2^{\circ}$ and $94.54^{\circ}$ for the collimated and surface detectors, respectively.

\section{DATA PRODUCTS}

Each FIREBIRD unit produces a context and high resolution (hi-res) data product. Hi-res data are the primary data product and

TABLE II. Energy ranges of the FIREBIRD detectors $(\mathrm{keV})$ and median geometric factors $\left(\mathrm{cm}^{2} \mathrm{sr}\right)$ during campaigns 21-24. The minimum energy was reduced to about $200 \mathrm{keV}$, and the first five energy channels were compressed to study the low energy electrons in finer detail. The surface detector on FU4 has not functioned since launch, so its energy ranges are excluded. The response of the surface detector on FU3 changed significantly long before these campaigns, so its energy ranges are also excluded.

\begin{tabular}{lcccc}
\hline \hline \multicolumn{2}{c}{ FU3 Collimated } & & \multicolumn{2}{c}{ FU4 Collimated } \\
Energy range $(\mathrm{keV})$ & G factor $\left(\mathrm{cm}^{2} \mathrm{sr}\right)$ & & Energy range $(\mathrm{keV})$ & G factor $\left(\mathrm{cm}^{2} \mathrm{sr}\right)$ \\
\hline $201.2-250.2$ & 4.9 & & $201.0-246.5$ & 4.9 \\
$250.2-299.2$ & 5.1 & & $246.5-301.1$ & 5.3 \\
$299.2-348.2$ & 5.0 & $301.1-346.6$ & 4.9 \\
$348.2-446.2$ & 5.9 & $346.6-446.7$ & 5.9 \\
$446.2-1055.2$ & 6.6 & & $446.7-983.6$ & 6.5 \\
$>1055.2$ & 3.6 & $>983.6$ & 4.2 \\
\hline \hline
\end{tabular}


TABLE III. Energy channel sampled by context channel 1 for each campaign.

\begin{tabular}{lllll}
\hline \hline & FU3 & & \multicolumn{2}{c}{ FU4 } \\
\cline { 1 - 1 } \cline { 5 - 6 } Campaign & D1 channel (keV) & & Campaign & D1 channel (keV) \\
\hline $1-7$ & Surface 683-950 & & 1 & Surface 803-1104 \\
& & & $2-7$ & Collimated 283-384 \\
$8-9$ & Collimated 231-300 & & $8-9$ & Collimated 220-283 \\
$10-20$ & Collimated 300-408 & & $10+$ & Collimated 283-384 \\
$21-24$ & Collimated 299-348 & & $21-24$ & Collimated 301-347 \\
\hline \hline
\end{tabular}

consist of six energy channels logarithmically spaced from about $200 \mathrm{keV}$ to $>1 \mathrm{MeV}$ at high time cadence for both collimated and surface detectors. The energy range of each hi-res channel is shown in Table I for campaigns 1-20 and Table II for campaigns $21+$. The time cadence can be found in column 3 in Table IV. Due to limited bandwidth, less than $1 \%$ of hi-res data are downloaded each campaign, and the rest is discarded (see Sec. VII). Hi-res data to be downloaded are selected by a scientist-in-the-loop using the context data to estimate times of microburst activity or other interesting precipitation. Other factors such as geomagnetic activity and conjunctions with other missions will also inform this selection. Typically, a radiation belt pass will be downloaded from both spacecraft, which allows comparison of the electron activity on time scales of minutes.

Context data consist of two selected hi-res channels at a $6 \mathrm{~s}$ cadence and is fully downloaded each campaign. Context channel 0 has always sampled the collimated high energy integral channel on both flight units, $>1055.2 \mathrm{keV}$ on FU3 and $>985.0 \mathrm{keV}$ on FU4. Context channel 1 has changed several times over the course of the mission. It originally sampled the lowest energy surface channel; however, this was changed to the second lowest energy collimated channel on FU4 starting in campaign 2 since the surface detector was not functioning. Starting in campaign 8 , channel 1 was changed to the lowest energy collimated channel on both spacecraft for consistency between the two flight units. In campaign 10, both were raised to the second lowest collimated channel. Starting in campaign 21, the hi-res energy channels were changed to better investigate lower energies causing another shift in context channel 1 while the integral channel, and thus context 0 , remained the same. The sampling for context channel 1 is summarized in Table III.

\section{CAMPAIGN STRUCTURE}

FIREBIRD was designed with a philosophy of "fewer events at greater detail." ${ }^{\prime 1}$ The high cadence and energy resolution produces far more data than can be downloaded in a practical amount of time, so a campaign strategy has been developed. FIREBIRD takes data continuously until its memory has been filled, which typically takes 3-6 weeks depending on the settings chosen for the campaign. Section VII A describes the various settings that have been used. Once memory is filled, the instrument is turned off to prevent overwriting while data continue to be downloaded until the science team determines a new campaign should be started. Typical reasons for beginning a new campaign are upcoming conjunction opportunities, such as the 2015 and 2016 BARREL campaigns, or the completion of hi-res downloads relevant to the campaign's science objective.

Table IV summarizes the FIREBIRD data campaigns completed to date. The early mission focused on the collection of microbursts, while the separation between the spacecraft remained small. After the first campaign, the spacecraft had separated well beyond the expected scale size of a microburst preventing any further self-coincident observations, so the focus began to turn toward conjunctions with other missions. Hi-res downloads now focus almost exclusively on conjunctions with missions such as the Van Allen Probes, Arase, ${ }^{46}$ and BARREL so that the majority of hires data from recent campaigns has at least one other mission in conjunction.

The typical operating cadence has also been changed from $18.75 \mathrm{~ms}$ to $50 \mathrm{~ms}$ to improve download capabilities. The original cadence of $18.75 \mathrm{~ms}$ was chosen to mimic the highest SAMPEX resolution of $20 \mathrm{~ms}$; however, microbursts can still be resolved at $50 \mathrm{~ms}$ while allowing a greater portion of the hi-res data to be downloaded. Decreasing the hi-res cadence does not increase the length of a campaign due to the context partition in spacecraft memory filling at the same rate as before.

The spacecraft separation is shown in Fig. 7. The separation is almost entirely along the orbital path with a negligible cross-track separation. The black line shows the total separation spatially (left hand axis) and temporally (right hand axis) with the blue dots at the top representing the start of each data campaign. The spacecraft have steadily separated since launch with the exception of a few months at the end of 2015. Any variation in separation is due to changes in the drag profile caused by a different attitude or rotation of the spacecraft. There is no active control over spacecraft pointing, nor any information on spacecraft pointing, so the precise attitude and rotation is unknown.

\section{A. Campaign parameters}

FIREBIRD has several campaign parameters that can be changed on-orbit, the most important of which are hi-res cadence and fraction of orbit to take data. The hi-res data cadence can be set to multiples of $6.25 \mathrm{~ms}$, up to a maximum of $100 \mathrm{~ms}$. A data rate of $6.25 \mathrm{~ms}$ can be selected; however, the data are produced too fast to be recorded effectively, making $12.5 \mathrm{~ms}$ the fastest possible cadence. In addition, FIREBIRD can be set to take data over a full orbit or can be restricted to taking data for only part of an orbit in order to lengthen the campaign. Due to restrictions on how data are written to memory, it is most efficient to choose either half or full orbits.

Additional parameters of interest are the selection of hi-res channels to sample for context data and the boundaries of the hires energy channels. The hi-res energy channels sampled for context data are summarized on Table III. The boundaries of the hi-res energy channels can be set to any of the 256 discrete values between $0 \mathrm{MeV}$ and $\sim 2 \mathrm{MeV}$; however, the detectors can only fully absorb electrons up to $1050 \mathrm{keV}$ (Sec. III), making a selection above $\sim 1 \mathrm{MeV}$ impractical. Table I describes the energy boundaries that were used for campaigns 1-20. Starting in campaign 21, the energy channels were shifted to explore the low energy spectrum in greater detail and are described in Table II. 
TABLE IV. Summary of FIREBIRD data campaigns. The first campaign was focused on finding microbursts simultaneously observed on both spacecraft. After the first campaign, the spacecraft had separated beyond the expected scale size of a microburst, so the focus switched to conjunctions with other magnetospheric missions.

\begin{tabular}{|c|c|c|c|}
\hline Campaign & Dates & Cadence (ms) & Primary science \\
\hline 1 & $2015 / 2 / 1-2015 / 2 / 21$ & 18.75 & Spatial scale of individual microbursts \\
\hline 2 & $2015 / 3 / 21-2015 / 4 / 19$ & 18.75 & St. Patrick's Day storm \\
\hline 3 & $2015 / 5 / 16-2015 / 6 / 15$ & 18.75 & Van Allen Probes conjunctions \\
\hline 4 & $2015 / 7 / 3-2015 / 8 / 4$ & 18.75 & July 4 th storm \\
\hline 5 & $2015 / 8 / 8-2015 / 9 / 4$ & 18.75 & BARREL conjunctions \\
\hline 6 & 2015/11/15-2015/12/15 & 18.75 & Lightning induced precipitation \\
\hline 7 & $2016 / 1 / 15-2016 / 2 / 3$ & 12.5 & GRIPS $^{\mathrm{a}}{ }^{42}$ conjunctions \\
\hline 8 & $\begin{array}{l}\text { FU3: } 2016 / 5 / 20-2016 / 6 / 14 \\
\text { FU4: } 2016 / 6 / 9-2016 / 6 / 20\end{array}$ & 50 & $\operatorname{COSI}^{\mathrm{b}} 43$ conjunctions and staggered operation \\
\hline 9 & $2016 / 8 / 12-2016 / 9 / 7$ & 50 & BARREL conjunctions \\
\hline 10 & $2016 / 12 / 21-2017 / 1 / 4$ & 12.5 & Time of flight dispersion \\
\hline 11 & $2017 / 5 / 1-2017 / 5 / 21$ & 50 & Multi-mission conjunction on May 2nd \\
\hline 12 & $2017 / 7 / 1-2017 / 7 / 21$ & 50 & Van Allen Probes and Arase conjunctions \\
\hline 13 & $2017 / 11 / 19-2017 / 12 / 14$ & 50 & Van Allen Probes conjunctions \\
\hline 14 & $2018 / 2 / 27-2018 / 3 / 28$ & 50 & Van Allen Probes and MMS ${ }^{44}$ conjunctions \\
\hline 15 & $2018 / 4 / 20-2018 / 5 / 13$ & 50 & AC6 lapping events \\
\hline 16 & $2018 / 6 / 25-2018 / 7 / 18$ & 50 & BARREL piggyback conjunctions \\
\hline 17 & $2018 / 7 / 31-2018 / 8 / 20$ & 50 & POES ${ }^{c}$, Van Allen Probes, PFISR, and AC6 conjunctions \\
\hline 18 & $2018 / 9 / 17-2018 / 10 / 13$ & 50 & POES, Van Allen Probes, PFISR, and AC6 conjunctions \\
\hline 19 & $2018 / 12 / 16-2019 / 1 / 10$ & 50 & BARREL super pressure conjunctions \\
\hline 20 & $2019 / 1 / 24-2019 / 2 / 20$ & 50 & BARREL super pressure conjunctions (continued) \\
\hline 21 & $2019 / 3 / 16-2019 / 4 / 10$ & 50 & Modified energy channels (see Table II) \\
\hline 22 & $2019 / 5 / 5-2019 / 5 / 17$ & 12.5 & Dispersion study with new energy channels \\
\hline 23 & $2019 / 7 / 5-2019 / 7 / 29$ & 50 & Van Allen Probes conjunctions \\
\hline 24 & 2019/9/10-2019/10/8 & 50 & Van Allen Probes and DSX ${ }^{\mathrm{d}} 45$ conjunctions \\
\hline 25 & $2020 / 1 / 2-2020 / 1 / 27$ & 50 & BARREL super pressure conjunctions, FU4 only \\
\hline
\end{tabular}

${ }^{a}$ Gamma ray imager/polarimeter for solar flares.

${ }^{\mathrm{b}}$ Compton spectrometer and imager.

${ }^{\mathrm{c}}$ Polar operational environmental satellites.

${ }^{\mathrm{d}}$ Demonstration and science experiments.

\section{DATA PROCESSING}

FIREBIRD data are downloaded at a ground station hosted by Montana State University in Bozeman, MT. Signal processing is handled by a software-defined radio built in GNU Radio, and spacecraft commanding and raw data management are handled by mission unique FIREBIRD scripts operating under L3's InControl Satellite Command and Control Software package. At the beginning of the mission, passes were run manually by student volunteers whose primary job was to monitor the incoming data and determine if there were any packets lost in the transmission that needed to be re-downloaded. This method left many passes unoperated, such as those happening on weekends or at night, which reduced the amount of data that could be downloaded. During the summer of 2018, an automated script was developed to operate passes. This script greatly increased the amount of data that could be downloaded, by both operating passes that would not be otherwise manned and increasing the efficiency of each individual pass.

After each pass, data are exported from InControl and uploaded to a local server, where geographic and magnetic ephemerides are added to the data. In addition, the processed data include a time stamp correction for the count rates, expressed as a number of seconds to be added to the time stamps. A brief description of this correction is given in Sec. VIII A, and a detailed description of the calculation can be found in the Appendix. This time correction is typically calculated a few days after the spacecraft memory is filled for the campaign and may not yet be available until after the most recent campaign is completed. The ephemeris data are calculated on the ground (described below) and, therefore, does not need a time correction.

Geographic ephemeris is calculated by propagating the most recent Two Line Element (TLE) using the SGP-4 (Simplified General Perturbations) algorithm. ${ }^{47}$ The SGP-4 algorithm uses these TLEs to calculate the orbital state vectors accounting for perturbations such as Earth's atmosphere and gravitational effects from the moon and sun. ${ }^{47}$ The generated geographic ephemeris data are used to calculate the Mcllwain $\mathrm{L}^{48}$ and MLT of the spacecraft using the Tsyganenko 1989 magnetic field model ${ }^{49}$ and real time Kp.

The hi-res data file also includes an electron flux value for each energy channel in addition to the count rates. Flux rates are 


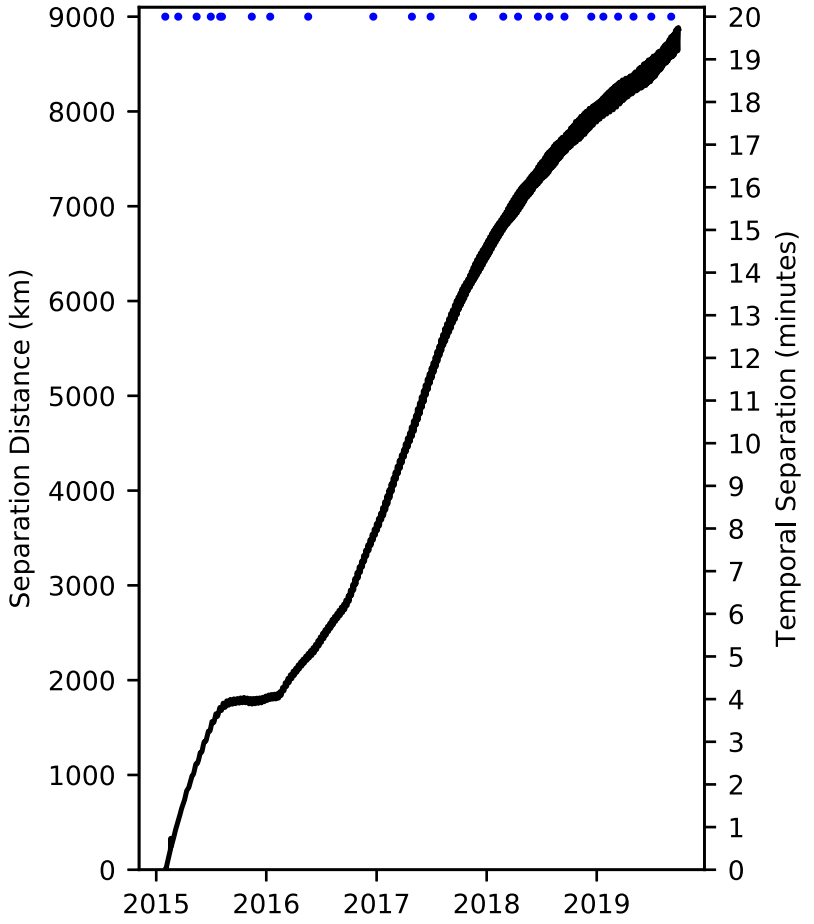

FIG. 7. Total separation between FU3 and FU4 from launch to the end of September 2019. The width of the line represents the variation in spacecraft separation over a single orbit. The blue dots at the top show the start of each data campaign.

calculated by dividing by channel bin width, cadence, and analytic geometric factor, $9 \mathrm{~cm}^{2}$ sr for the collimated detector and $23 \mathrm{~cm}^{2} \mathrm{sr}$ for the surface detector. Modeling done in GEANT-4 has revealed the effective geometric factors to be closer to $6 \mathrm{~cm}^{2}$ sr and $14 \mathrm{~cm}^{2} \mathrm{sr}$ for the collimated and surface detectors, respectively (see Sec. V). As a first order correction, the fluxes found in the FIREBIRD data product should be increased by $50 \%$ for the collimated detector and $60 \%$ for the surface detector. A more precise correction can be performed using the full GEANT data set as described in Sec. V.

The final data product is released using the JavaScript Object Notation (JSON) headed ASCII format. The JSON head includes meta-data such as column names, descriptions, and units, which can be read in along with the data. The data are white space delimited and can be read in independently of the JSON header, if desired. The data are uploaded daily to http://solar.physics.montana.edu/FIREBIRD_II/.

\section{A. Time correction}

The clocks on FIREBIRD are synced to GPS time each time a GPS lock is obtained but are otherwise allowed to drift freely with a measured drift on the order of $1 \mathrm{~s} /$ day. Due to power budget constraints, the GPS is turned on for a single orbit each day and attempts to obtain a lock. If successful, the on-board clock will be adjusted to the current GPS time and the GPS unit will be turned off again. The GPS connection is not successful every day, with FU3 being successful most days but FU4 often going weeks without a successful connection. In addition, when the clock is adjusted, the time and magnitude of the adjustment are not recorded. These factors mean there is insufficient information to fully characterize this drift; so, a method of approximating the drift had to be developed. A brief description of this correction is described here, and additional detail can be found in the Appendix. Since the ephemeris data are calculated separately using the TLEs, only the count data need to be corrected.

The only information available to quantify the FIREBIRD clock drift is contained in the telemetry packets (hereafter beacons) that FIREBIRD sends every $30 \mathrm{~s}$ during standard operation. These beacons contain engineering data such as temperatures and voltages as well as a time stamp from the FIREBIRD clock. These beacons are received and recorded at the MSU ground station during each pass of the satellites. The beacon time stamp can be compared to the ground station clock to determine the relative time difference between the FIREBIRD and ground clocks (referred to as $\Delta t_{b}$ ) and tracked over time to determine a relative drift (the FIREBIRD-ground drift). Early in the mission, the ground station clock was infrequently synced to UTC time, resulting in a second source of drift between FIREBIRD and UTC (the ground-UTC drift).

Both FIREBIRD-ground drift and ground-UTC drift can be estimated using the time stamps on the received beacons. When a large change in $\Delta t_{b}$ is observed between subsequent beacons, it is inferred that one of the clocks was synced. If this change is observed on just one flight unit, the sync must have occurred on the FIREBIRD clock, but if both units observe a similar change in $\Delta t_{b}$, it is likely that the ground clock was synced. After a ground clock sync, the ground station is returned to agreement with UTC, and the change in $\Delta t_{b}$ must correspond to the adjustment applied. The ground-UTC drift is linearly modeled and used to correct the ground time to yield the approximate difference between FIREBIRD and UTC. Using the corrected ground time, the FIREBIRD-UTC drift is approximated with a linear fit that can be applied to the FIREBIRD data.

A few improvements have been made to reduce ambiguity in the time correction as the problem was characterized. Beginning in campaign 8 , the Internet Time Service (ITS) provided by the National Institute of Standards and Technology (NIST) was implemented on the ground station. The ITS uses the Network Time Protocol (NTP) to sync the ground clock every hour and records the time and magnitude of any adjustment to a log. The hourly correction is typically on the order of 10 s of $\mathrm{ms}$ and can be neglected. A second improvement was to turn the GPS off completely during campaigns beginning in campaign 10. Each day, FIREBIRD passes over the MSU ground station 3-5 times, and due to the manual operation of FIREBIRD in the early and middle missions, there are many days with some or all passes missing. With daily GPS syncs, the beacons must be split into daily chunks with only a few data points, increasing ambiguity. By turning GPS off, every beacon during the campaign, typically over 100 , can be used in the fit. In addition, the antenna tracking of FIREBIRD was automated in the summer of 2017 making beacons available for every pass, even those not operated. With these improvements, the ground clock is known to be within 10s of ms, more beacons are available to model, and the beacon data are uninterrupted across the campaign. 
FU3 Hi-Res February 1, 2015

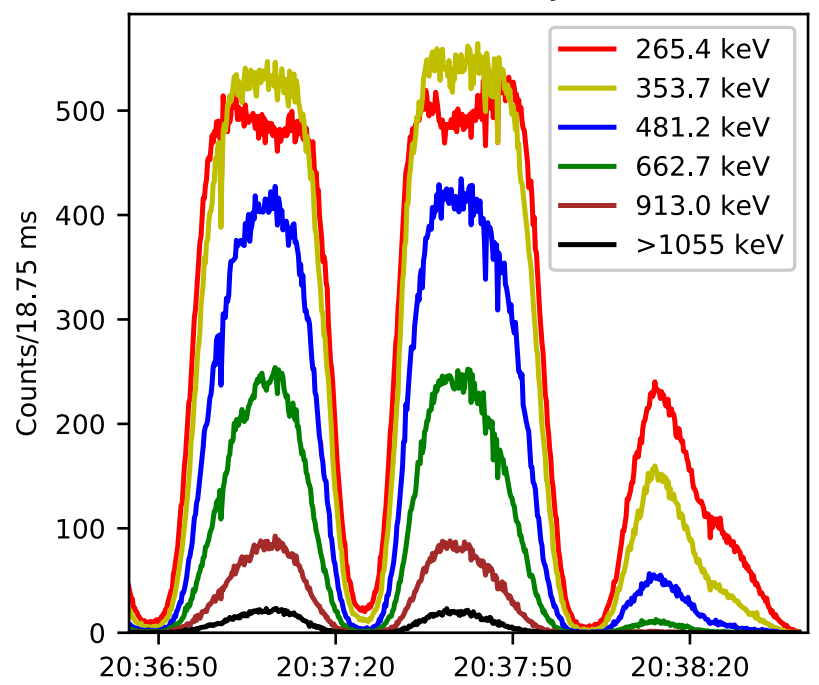

FIG. 8. FU3 hi-res data from campaign 1 . The periodic increase in flux is attributed to oscillations in spacecraft pointing causing different pitch angles to be sampled. Effects of detector saturation can be seen at the first two peaks where the second energy channel rises above the first. The data have been smoothed by averaging over every 10 data points.

\section{OTHER CONSIDERATIONS}

FIREBIRD is equipped with a passive alignment system using a permanent magnet and two hysteresis rods designed to align the detectors with the magnetic field. Unfortunately, this system was not able to stabilize the spacecraft pointing along the magnetic field, and during the first several campaigns, an oscillation is seen in the data with a period of about $40 \mathrm{~s}$ as the spacecraft samples different pitch angles. An example of this can be seen in Fig. 8. It is expected that the higher count rates are a result of sampling more of the trapped population, but the precise pointing is unknown, preventing a quantitative analysis. In later campaigns, this oscillation is not always observed in the data, but it continues to be observed in the signal strength at the ground station and will still occasionally be observed in the data.

During periods of high electron flux instrument saturation effects become apparent. The effects of saturation can be seen in Fig. 8 as a flattening of the peaks and the higher count rates in the second energy channel compared to the first. While the DAPPER chip is processing the signal from instrument detection, it will not accept additional detections causing them to be dropped. During periods of high flux electrons will strike the detector at a rate faster than the DAPPER can process them resulting in a cap on the flux value that can be recorded. Each detection event requires a different amount of time to process, scaling linearly with energy deposited, causing this cap flux value to depend on the energy spectrum and to be especially noticeable during periods of high energy flux.

\section{SUMMARY}

The success of FIREBIRD-II can be largely attributed to good design, sensible risk management, and effective use of limited resources. The design was a mixture of purchased COTS components when suitable and custom components when an appropriate COTS option could not be found. A focus on simple solutions, such as the replacement EPS board, reduced the number of potential failure points. The budget and size of CubeSat missions does not allow for redundant systems, so every potential failure point can be mission ending. The FIREBIRD mission objectives were carefully selected to have high science value while being achievable within the limitations of a CubeSat mission. The CMDSEQ functionality in the operating system not only allows flexibility in the operating parameters but also provides an avenue for software fixes on orbit such as disabling GPS during data runs to improve timing corrections. The WDT on the EPS board resolves software latch ups without ground intervention and has saved the mission more than once when the spacecraft became unresponsive to ground commands.

The FIREBIRD mission has been highly successful and has far exceeded its expected mission lifetime of 120 days, corresponding to just the first three campaigns. Simultaneous microbursts were observed on each unit within a day of launch allowing for direct measurements of the scale size. ${ }^{24,25}$ FIREBIRD observations of a microburst region combined with AC6 and BARREL provided the spatial scale and duration of that region, ${ }^{27}$ improving our understanding of electron loss due to microburst precipitation. FIREBIRD has also enabled additional science beyond the original scope of the mission, including a direct link between chorus waves observed on Van Allen Probes A and microbursts observed on FIREBIRD. ${ }^{34}$ Additional ongoing studies into the microburst energy spectrum, EMIC scattered electron precipitation, the effects of microbursts on atmospheric chemistry, and the signature of microbursts when viewed from ground based radar continue to expand the scope and contributions of the FIREBIRD mission.

\section{ACKNOWLEDGMENTS}

The FIREBIRD mission is supported by the National Science Foundation under Grant Nos. 0838034, 1339414, and 1035642. A. T. Johnson was supported by Montana State University under startup funding for J. Sample. M. Shumko was supported by the National Aeronautics and Space Administration (NASA) Headquarters under the NASA Earth and Space Science Fellowship Program, Grant No. 80NSSC18K1204. The authors acknowledge Phillip Ward for his work automating the ground station at MSU as a Research Experience for Undergraduates student during the summer of 2018. In addition, the authors acknowledge any student who volunteered time to operate FIREBIRD passes and the members of the Space Science and Engineering Laboratory at Montana State University for their hard work making FIREBIRD a success. FIREBIRD-II data are uploaded daily to http://solar.physics.montana.edu/FIREBIRD_II/.

\section{APPENDIX: TIME CORRECTION METHODOLOGY}

The time stamping of FIREBIRD data are governed by two separate Real Time Clocks (RTC) on the Multi-Function Interface Board (MFIB) and Command and Data Handling (CDH) boards (see Sec. IV). There is insufficient information to fully characterize the drift of these clocks, so a method of approximating a time correction for this drift has been developed and is described here. This method is intended as a first order correction that can automatically be applied to each FIREBIRD campaign. More sophisticated 
techniques might be able to improve the accuracy of the estimate, but these must be considered on a case by case basis making them unsuitable for an automatic blanket correction. An improved estimate can be made by the FIREBIRD team on request. Ephemeris data are calculated independently of the FIREBIRD system (see Sec. VIII) and do not need to be corrected; the correction described here only applies to the count and flux data.

The MFIB microcontroller runs software RTC which is responsible for time stamping the data. At the beginning of each data run, the MFIB RTC is synced to the CDH RTC but is not compared at any other time, making a direct estimate of drift impossible. Attempts have been made to compare the length of a data run as specified by the onboard command sequences to the length according to the data; however, the spacecraft is allowed up to $30 \mathrm{~s}$ to execute the data run start/stop command, resulting in an error much larger than the expected drift. The clock is synced at least once per orbit, allowing at most one orbital period to drift ( $\sim 95 \mathrm{~min})$. Based on the clock data sheet, the accuracy after 5 years should be within 25 ppm, which corresponds to about $0.135 \mathrm{~s} /$ orbit in the worst case.

The $\mathrm{CDH}$ has hardware RTC with many roles in addition to syncing the MFIB clock, including time stamping a pair of telemetry packets (hereafter beacons) which are broadcast every $30 \mathrm{~s}$. These beacons are collected by the ground station during passes. By comparing the beacon time stamp to the ground station time, a relative drift can be estimated. We define the difference between the ground clock and CDH RTC as $\Delta t_{b}=t_{g}-t_{c}$, where $t_{g}$ is the ground clock time when a beacon is received and $t_{c}$ is the CDH time stamp of the beacon. Figure 9 (a) shows the uncorrected $\Delta t_{b}$ for campaign 6 , and
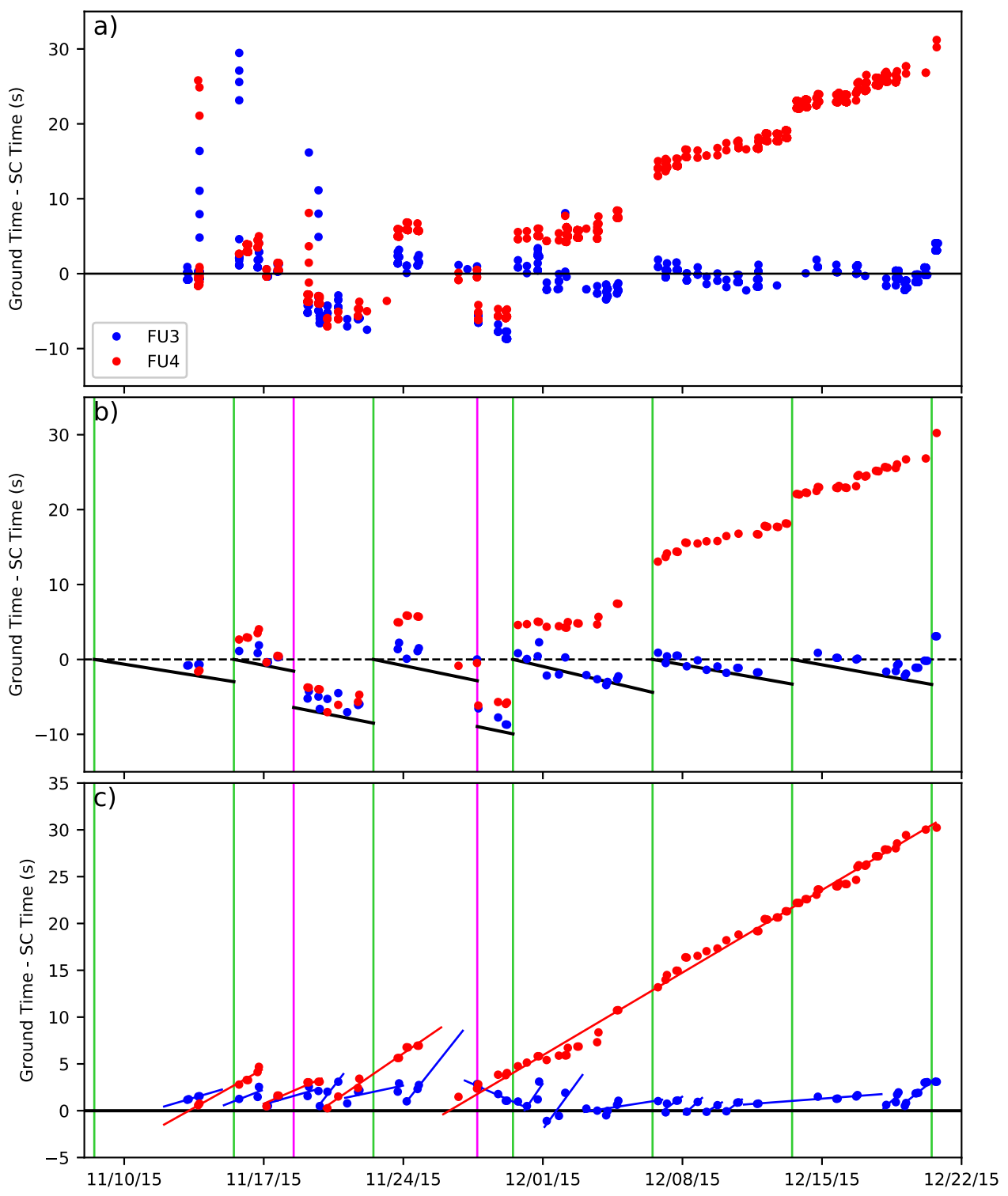

FIG. 9. Beacon information from campaign 6 illustrating the effects of each time correction. (a) Uncorrected beacon data. (b) Beacon data after removing delayed beacons and showing the estimated correction to the ground clock drift as solid black lines. Vertical green lines indicate a time of likely ground sync, and vertical magenta lines indicate anomalous changes in $\Delta t_{b}$ for both flight units likely attributable to a change in the ground clock but not due to a sync, such as a power outage. (c) Beacon data with the ground fit subtracted off. These data are fit and reported in the FIREBIRD data product as a time correction. 
Figs. 9(b) and 9(c) demonstrate each of the corrections described here.

Under default settings, FIREBIRD will attempt to connect to GPS once per day. If a connection is not made within one orbit, the GPS is turned back off until the next day. When successful, a GPS lock counter is incremented, and the CDH clock is synced, but the time of the sync and amount the clock was adjusted are not recorded. In addition, the lock counter is susceptible to false positives, so additional information is needed to determine if the clock has been synced or not. Starting in campaign 10, the GPS has been turned off while data are being taken to remove these ambiguities.

The ground station clock is the computer clock on a Windows 7 machine running L3's InControl. During the first seven campaigns, it is uncertain how often this clock was synced; however, it was likely using the Window's default of once per week. In addition, the amount the clock was adjusted is unknown. Starting in campaign 8, the National Institute of Standards and Technology (NIST) Internet Time Service (ITS) has been used to manage the ground clock. This software syncs with NIST servers once per hour using the Network Time Protocol (NTP) and records when the clock was synced and how much it was adjusted. The hourly correction is typically on the order of $10 \mathrm{~s}$ of $\mathrm{ms}$ and can be neglected. In instances of a larger correction, the drift can easily be estimated using the log. At the beginning of campaign 23 , the ground station computer was replaced with a new machine running Linux Ubuntu 18.04 and the built in NTP is used to maintain clock accuracy in a similar way to the NIST ITS.

Before the drift corrections can be made, $\Delta t_{b}$ must be filtered because there is often a delay between the packaging and transmitting of a beacon. This delay will typically be approximately $1 \mathrm{~s}$; however delays of over $3 \mathrm{~s}$ have occurred. This will always be a positive error in $\Delta t_{b}$ and can be mitigated by only using the minimum $\Delta t_{b}$ from each pass. If only a single beacon was received during a pass, it cannot be determined if that beacon was delayed so that it is also removed from consideration. Figure 9(b) shows the beacons remaining after this filter.

For campaigns 1-7, the ground clock drift must be corrected. The time or magnitude of ground clock syncs is not known, so the drift must be inferred from the beacon data. If $\Delta t_{b}$ shows a large change between two passes, we can assume either the $\mathrm{CDH}$ or ground clock changed, likely due to sync. If this change is only observed on one flight unit, it is likely the $\mathrm{CDH}$ time that was synced with GPS, which can be verified by checking the GPS lock counter. If both flight units observe a similar change, it is likely the ground clock that was synced. Times of likely ground sync have been identified as vertical green lines in Fig. 9(b) and show a good agreement with the assumption of syncing once per week. The magnitude of the adjustment is approximated by averaging the change in $\Delta t_{b}$ for both flight units. The drift is assumed to be linear across the week, starting at 0 at the time of the most recent sync and reaching the calculated clock adjustment a week later. This is represented by the solid black lines in Fig. 9(b).

Occasionally, a change in $\Delta t_{b}$ will be observed on both flight units that break the pattern of weekly ground clock syncs and increase the difference between the ground clock and correct time. There are two examples of this in campaign 6 marked by vertical magenta lines in Fig. 9(b) on November 18 and 28. These are likely caused by a change in the ground clock time for reasons other than a sync, such as a power outage. The magnitude of the change is once again found by averaging the change in $\Delta t_{b}$ for both flight units and is treated as a constant offset for beacons afterward.

Once corrections to the ground clock have been determined, they can be subtracted from $\Delta t_{b}$ to get the estimated difference between the $\mathrm{CDH}$ clock and UTC time allowing the drift of the $\mathrm{CDH}$ clock to be analyzed directly. The beacons are first split into groups according to times the CDH clock synced to GPS. These times are identified by looking for an increment to the GPS lock counter coincident with $\Delta t_{b}$ returning to near zero. Once the beacons have been grouped, a linear fit is applied to estimate the $\mathrm{CDH}$ clock drift, shown in Fig. 9(c).

There are still a few challenges to fitting these groups of beacons. Each day, 3-5 passes will occur, and due to the manual operation of the FIREBIRD ground station during early campaigns, there are many days with some or all passes missing. With the GPS set to sync once per day, the clock drift is often underdefined, and some assumptions must be made. Figure 9(c) shows that FU3 has many more successful GPS syncs than FU4, which actually makes estimating the drift more difficult. For example, from December 11-18, very few beacons were collected on FU3 obscuring the actual clock drift. These beacons are grouped together for a fit, but it could be that one or more of these days had a GPS sync that is not accounted for. On days with zero beacons, a fit might have to be extrapolated from the previous or next day, such as FU3 on November 26-27. This extrapolation is only accurate if there was no GPS sync; however, there is no way to determine if that is the case. As mentioned earlier, the GPS is turned off while data are being taken starting with campaign 10 , allowing several weeks of beacons to be used in performing the correction fit and reducing the number of assumptions needed.

During campaign 14, the time stamps on FU4 were observed to occasionally be incorrect by a day or more. About halfway through the campaign, the year on the time stamps jumped to 2165 while the day of the year varies across about 8 months. Attempts were made to back out the correct time stamps for this period but were ultimately unsuccessful, resulting in a large amount of effectively lost data. This was corrected by turning the GPS on, and once corrected, the spacecraft resumed normal operation. The ambiguities associated with the GPS are preferable to the grossly inaccurate times observed in campaign 14, so the original strategy of daily GPS syncs has been used on FU4 starting in campaign 15. The ground station was updated to include automatic antenna tracking of the satellites in the summer of 2017, making beacons available for every pass and reducing the ambiguities of having GPS on during data collection.

\section{REFERENCES}

${ }^{1}$ H. E. Spence, J. B. Blake, A. B. Crew, S. Driscoll, D. M. Klumpar, B. A. Larsen, J. Legere, S. Longworth, E. Mosleh, T. P. Obrien, S. Smith, L. Springer, and M. Widholm, Space Weather 10, S11004, https://doi.org/10.1029/2012sw000869 (2012).

${ }^{2}$ D. Klumpar, L. Springer, E. Mosleh, K. Mashburn, S. Berardinelli, A. Gunderson, M. Handley, N. Ryhajlo, H. Spence, S. Smith, J. Legere, M. Widholm, S. Longworth, A. Crew, B. Larsen, J. B. Blake, and N. Walmsley, Proceedings of the 29th Annual AIAA/USU Conference on Small Satellites, 2015.

${ }^{3}$ K. A. Anderson and D. W. Milton, J. Geophys. Res. 69, 4457, https://doi.org/ 10.1029/jz069i021p04457 (1964).

${ }^{4}$ G. K. Parks, J. Geomagn. Geoelectr. 30, 327 (1978). 
${ }^{5}$ W. L. Imhof, H. D. Voss, J. Mobilia, D. W. Datlowe, E. E. Gaines, J. P. McGlennon, and U. S. Inan, J. Geophys. Res. 97, 13829, https://doi.org/10.1029/ 92ja01138 (1992).

${ }^{6}$ J. Blake, M. Looper, D. Baker, R. Nakamura, B. Klecker, and D. Hovestadt, Adv. Space Res. 18, 171 (1996).

${ }^{7}$ K. R. Lorentzen, J. B. Blake, U. S. Inan, and J. Bortnik, J. Geophys. Res. 106, 6017, https://doi.org/10.1029/2000ja003018 (2001).

${ }^{8}$ G. Parks, J. Geophys. Res. 72, 215, https://doi.org/10.1029/jz072i001p00215 (1967).

${ }^{9}$ S. Dietrich, C. J. Rodger, M. A. Clilverd, J. Bortnik, and T. Raita, J. Geophys. Res. 115, A12240, https://doi.org/10.1029/2010JA015777 (2010).

${ }^{10}$ R. M. Millan, R. P. Lin, D. M. Smith, K. R. Lorentzen, and M. P. McCarthy, Geophys. Res. Lett. 29, 47, https://doi.org/10.1029/2002gl015922 (2002).

${ }^{11}$ L. A. Woodger, A. J. Halford, R. M. Millan, M. P. McCarthy, D. M. Smith, G. S. Bowers, J. G. Sample, B. R. Anderson, and X. Liang, J. Geophys. Res.: Space Phys. 120, 4922, https://doi.org/10.1002/2014ja020874 (2015).

${ }^{12}$ K. R. Lorentzen, M. D. Looper, and J. B. Blake, Geophys. Res. Lett. 28, 2573, https://doi.org/10.1029/2001gl012926 (2001).

${ }^{13}$ T. P. O’Brien, M. D. Looper, and J. B. Blake, Geophys. Res. Lett. 31, L04802, https://doi.org/10.1029/2003GL018621 (2004).

${ }^{14}$ R. M. Thorne, T. P. O'Brien, Y. Y. Shprits, D. Summers, and R. B. Horne, J. Geophys. Res.: Space Phys. 110, A09202, https://doi.org/10.1029/2004JA010882 (2005).

${ }^{15}$ National Academies of Sciences, Engineering and Medicine, Achieving Science with CubeSats (The National Academies Press, Washington, DC, 2016).

${ }^{16} \mathrm{~J}$. G. Sample, R. M. Millan, and L. A. Woodger, in The Dynamic Loss of Earth's Radiation Belts (Elsevier, 2020), Chap. 5, pp. 121-144

${ }^{17} \mathrm{M}$. Wiltberger, Committee on Solar and Space Physics Spring Meeting, Washington, DC, 2018.

${ }^{18}$ D. Gerhardt, S. E. Palo, Q. Schiller, L. Blum, X. Li, and R. Kohnert, J. Small Satell. 3, 265 (2013).

${ }^{19}$ T. P. O'Brien, J. B. Blake, and J. W. Gangestad, AeroCube-6 Dosimeter Data README (v3.0), Technical Report No. TOR-2016-01155 Aerospace Corporation, El Segundo, CA, 2016.

${ }^{20}$ X. Li, R. Selesnick, Q. Schiller, K. Zhang, H. Zhao, D. N. Baker, and M. A. Temerin, Nature 552, 382 (2017).

${ }^{21}$ J. B. Blake and T. P. O’Brien, J. Geophys. Res.: Space Phys. 121, 3031, https://doi.org/10.1002/2015ja021815 (2016).

${ }^{22}$ S. Agostinelli, J. Allison, K. Amako, J. Apostolakis, H. Araujo, P. Arce, M. Asai, D. Axen, S. Banerjee, G. Barrand, F. Behner, L. Bellagamba, J. Boudreau, L. Broglia, A. Brunengo, H. Burkhardt, S. Chauvie, J. Chuma, R. Chytracek, G. Cooperman, G. Cosmo, P. Degtyarenko, A. Dell'Acqua, G. Depaola, D. Dietrich, R. Enami, A. Feliciello, C. Ferguson, H. Fesefeldt, G. Folger, F. Foppiano, A. Forti, S. Garelli, S. Giani, R. Giannitrapani, D. Gibin, J. J. Gomez Cadenas, I. Gonzalez, G. Gracia Abril, G. Greeniaus, W. Greiner, V. Grichine, A. Grossheim, S. Guatelli, P. Gumplinger, R. Hamatsu, K. Hashimoto, H. Hasui, A. Heikkinen, A. Howard, V. Ivanchenko, A. Johnson, F. W. Jones, J. Kallenbach, N. Kanaya, M. Kawabata, Y. Kawabata, M. Kawaguti, S. Kelner, P. Kent, A. Kimura, T. Kodama, R. Kokoulin, M. Kossov, H. Kurashige, E. Lamanna, T. Lampen, V. Lara, V. Lefebure, F. Lei, M. Liendl, W. Lockman, F. Longo, S. Magni, M. Maire, E. Medernach, K. Minamimoto, P. Mora de Freitas, Y. Morita, K. Murakami, M. Nagamatu, R. Nartallo, P. Nieminen, T. Nishimura, K. Ohtsubo, M. Okamura, S. O'Neale, Y. Oohata, K. Paech, J. Perl, A. Pfeiffer, M. G. Pia, F. Ranjard, A. Rybin, S. Sadilov, E. di Salvo, G. Santin, T. Sasaki, N. Savvas, Y. Sawada, S. Scherer, S. Sei, V. Sirotenko, D. Smith, N. Starkov, H. Stoecker, J. Sulkimo, M. Takahata, S. Tanaka, E. Tcherniaev, E. Safai Tehrani, M. Tropeano, P. Truscott, H. Uno, L. Urban, P. Urban, M. Verderi, A. Walkden, W. Wander, H. Weber, J. P. Wellisch, T. Wenaus, D. C. Williams, D. Wright, T. Yamada, H. Yoshida, and D. Zschiesche, Nucl. Instrum. Methods Phys. Res., Sect. A 506, 250 (2003); arXiv:1005.0727v1.

${ }^{23}$ J. Allison, K. Amako, J. Apostolakis, H. Araujo, P. A. Dubois, M. Asai, G. Barrand, R. Capra, S. Chauvie, R. Chytracek, G. A. Cirrone, G. Cooperman, G. Cosmo, G. Cuttone, G. G. Daquino, M. Donszelmann, M. Dressel, G. Folger, F. Foppiano, J. Generowicz, V. Grichine, S. Guatelli, P. Gumplinger, A. Heikkinen, I. Hrivnacova, A. Howard, S. Incerti, V. Ivanchenko, T. Johnson, F. Jones, T. Koi, R. Kokoulin, M. Kossov, H. Kurashige, V. Lara, S. Larsson, F. Lei, F. Longo,
M. Maire, A. Mantero, B. Mascialino, I. McLaren, P. M. Lorenzo, K. Minamimoto, K. Murakami, P. Nieminen, L. Pandola, S. Parlati, L. Peralta, J. Perl, A. Pfeiffer, M. G. Pia, A. Ribon, P. Rodrigues, G. Russo, S. Sadilov, G. Santin, T. Sasaki, D. Smith, N. Starkov, S. Tanaka, E. Tcherniaev, B. Tomé, A. Trindade, P. Truscott, L. Urban, M. Verderi, A. Walkden, J. P. Wellisch, D. C. Williams, D. Wright, H. Yoshida, and M. Peirgentili, IEEE Trans. Nucl. Sci. 53, 270 (2006).

${ }^{24}$ A. B. Crew, H. E. Spence, J. B. Blake, D. M. Klumpar, B. A. Larsen, T. P. O’Brien, S. Driscoll, M. Handley, J. Legere, S. Longworth, K. Mashburn, E. Mosleh, N. Ryhajlo, S. Smith, L. Springer, and M. Widholm, J. Geophys. Res.: Space Phys. 121, 5272, https://doi.org/10.1002/2016ja022485 (2016).

${ }^{25}$ M. Shumko, J. Sample, A. Johnson, J. B. Blake, A. Crew, H. Spence, D. Klumpar, O. Agapitov, and M. Handley, Geophys. Res. Lett. 45, 8811, https://doi.org/10.1029/2018gl078925 (2018).

${ }^{26}$ R. M. Millan, M. P. McCarthy, J. G. Sample, D. M. Smith, L. D. Thompson, D. G. McGaw, L. A. Woodger, J. G. Hewitt, M. D. Comess, K. B. Yando, A. X. Liang, B. A. Anderson, N. R. Knezek, W. Z. Rexroad, J. M. Scheiman, G. S. Bowers, A. J. Halford, A. B. Collier, M. A. Clilverd, R. P. Lin, and M. K. Hudson, Van Allen Probes Mission 9781489974, 503 (2013).

${ }^{27}$ B. R. Anderson, S. Shekhar, R. M. Millan, A. B. Crew, H. E. Spence, D. M. Klumpar, J. B. Blake, T. P. O’Brien, and D. L. Turner, J. Geophys. Res.: Space Phys. 122, 5949 (2017).

${ }^{28}$ R. Nakamura, M. Isowa, Y. Kamide, D. N. Baker, J. B. Blake, and M. Looper, J. Geophys. Res.: Space Phys. 105, 15875, https://doi.org/10.1029/2000ja900018 (2000).

${ }^{29}$ T. P. O’Brien, K. R. Lorentzen, I. R. Mann, N. P. Meredith, J. B. Blake, J. F. Fennell, M. D. Looper, D. K. Milling, and R. R. Anderson, J. Geophys. Res.: Space Phys. 108, 1329, https://doi.org/10.1029/2002JA009784 (2003).

${ }^{30}$ M. N. Oliven and D. A. Gurnett, J. Geophys. Res. 73, 2355, https://doi.org/ 10.1029/ja073i007p02355 (1968).

${ }^{31}$ T. J. Rosenberg, R. A. Helliwell, and J. P. Katsufrakis, J. Geophys. Res. 76, 8445, https://doi.org/10.1029/ja076i034p08445 (1971).

${ }^{32}$ T. J. Rosenberg, J. C. Siren, D. L. Matthews, K. Marthinsen, J. A. Holtet, A. Egeland, D. L. Carpenter, and R. A. Helliwell, J. Geophys. Res. 86, 5819, https://doi.org/10.1029/ja086ia07p05819 (1981).

${ }^{33}$ A. Y. Ukhorskiy, B. H. Mauk, N. J. Fox, D. G. Sibeck, and J. M. Grebowsky, J. Atmos. Sol.-Terr. Phys. 73, 1417 (2011).

${ }^{34}$ A. W. Breneman, A. Crew, J. Sample, D. Klumpar, A. Johnson, O. Agapitov, M. Shumko, D. L. Turner, O. Santolik, J. R. Wygant, C. A. Cattell, S. Thaller, B. Blake, H. Spence, and C. A. Kletzing, Geophys. Res. Lett. 44, 11265, https://doi.org/10.1002/2017gl075001 (2017).

${ }^{35}$ L. Capannolo, W. Li, Q. Ma, L. Chen, X. C. Shen, H. E. Spence, J. Sample, A. Johnson, M. Shumko, D. M. Klumpar, and R. J. Redmon, Geophys. Res. Lett. 46, 12711, https://doi.org/10.1029/2019gl084202 (2019).

${ }^{36}$ M. J. Nicolls and C. J. Heinselman, Geophys. Res. Lett. 34, L21104, https://doi.org/10.1029/2007GL031506 (2007).

${ }^{37}$ J. B. Blake, B. H. Mauk, D. N. Baker, P. Carranza, J. H. Clemmons, J. Craft, W. R. Crain, A. Crew, Y. Dotan, J. F. Fennell, R. H. Friedel, L. M. Friesen, F. Fuentes, R. Galvan, C. Ibscher, A. Jaynes, N. Katz, M. Lalic, A. Y. Lin, D. M. Mabry, T. Nguyen, C. Pancratz, M. Redding, G. D. Reeves, S. Smith, H. E. Spence, and J. Westlake, Space Sci. Rev. 199, 309 (2016).

${ }^{38}$ D. M. Klumpar, AIAA/USU Conference on Small Satellites (AIAA, 2012), p. SSC12.

${ }^{39}$ L. Lönnblad, Comput. Phys. Commun. 84, 307 (1994).

${ }^{40}$ K. Yando, R. M. Millan, J. C. Green, and D. S. Evans, J. Geophys. Res.: Space Phys. 116, A10231, https://doi.org/10.1029/2011ja016671 (2011).

${ }^{41}$ A. B. Crew, "Global distribution of microbursts in the Earth's magnetosphere," Ph.D. thesis, University of New Hampshire, 2013.

${ }^{42}$ A. Y. Shih, R. P. Lin, G. J. Hurford, N. A. Duncan, P. Saint-Hilaire, H. M. Bain, S. E. Boggs, A. C. Zoglauer, D. M. Smith, H. Tajima, M. S. Amman, and T. Takahashi, Space Telesc. Instrum. 2012: Ultraviolet Gamma Ray 8443, 84434H (2012).

${ }^{43}$ J.-L. Chiu, S. E. Boggs, H.-K. Chang, J. A. Tomsick, A. Zoglauer, M. Amman, Y.-H. Chang, Y. Chou, P. Jean, C. Kierans, C.-H. Lin, A. Lowell, J.-R. Shang, C.-H. Tseng, P. Von Ballmoos, and C.-Y. Yang, Nucl. Instrum. Methods Phys. Res., Sect. A 784, 359 (2015). 
${ }^{44}$ J. L. Burch, T. E. Moore, R. B. Torbert, and B. L. Giles, Space Sci. Rev. 199, 5 (2016).

${ }^{45}$ G. Spanjers, J. Winter, D. Cohen, A. Adler, J. Guarnieri, M. Tolliver, G. Ginet, B. Dichter, and J. Summers, in IEEE Aerospace Conference Proceedings 2006 (IEEE, 2006), p. 10

${ }^{46}$ Y. Miyoshi, Y. Kasaba, I. Shinohara, T. Takashima, K. Asamura, H. Matsumoto, N. Higashio, T. Mitani, S. Kasahara, S. Yokota, S. Wang, Y. Kazama, Y. Kasahara,
S. Yagitani, A. Matsuoka, H. Kojima, Y. Katoh, K. Shiokawa, K. Seki, M. Fujimoto, T. Ono, and ERG project Group, J. Phys.: Conf. Ser. 869, 012095 (2017).

${ }^{47}$ F. R. Hoots and R. L. Roehrich, Spacetrack Report No. 3, 1980.

${ }^{48}$ C. McIlwain, J. Geophys. Res. 66, 3681, https://doi.org/10.1029/jz066i011p03681 (1961).

${ }^{49}$ N. A. Tsyganenko, Planet. Space Sci. 37, 5 (1989). 